\title{
Relativistic density functional theory in nuclear physics
}

\author{
Jie Meng ${ }^{* \dagger}$ and Pengwei Zhao ${ }^{\dagger}$
}

\begin{abstract}
Over the past decades, the relativistic density functional theory has been greatly developed and widely applied to investigate a variety of nuclear phenomena. In this paper, we briefly review the concept of covariant density functional theory in nuclear physics with a few latest applications in describing nuclear ground-state and excitation properties as well as nuclear dynamics. Moreover, attempts to build a microscopic and universal density functional are also discussed in terms of the successful fully self-consistent relativistic Brueckner-Hartree-Fock calculations.
\end{abstract}

\section{Introduction}

During the past decades, the development of worldwide rare isotope beam facilities has extended our knowledge of nuclear physics from the stable to the unstable nuclei. Many unexpected features and novel phenomena have been found in neutron-rich nuclei. On the theoretical side, nuclear density functional theories (DFTs) have played important roles in the microscopic description of nuclei and the understanding of the new findings. In particular, the relativistic or covariant version of DFTs has attracted a lot of attentions due to many advantages. The development of covariant density functional theory (CDFT) and its applications in both ground-state and excitation properties have been introduced in detail in Ref. [1].

For a full understanding of nuclear systems, one has to solve, in principle, the nuclear many-body problem in a microscopic way. Nevertheless, a direct solution to such a many-body problem is very difficult if not impossible, in particular for heavy nuclei. The ab initio methods start from high precision realistic two-nucleon and/or threenucleon interactions and solve directly the nuclear manybody Hamiltonian [2, 3]. Usually, such methods are only accessible for very light nuclei due to the huge numerical costs. Configuration interaction shell models [4] build the nuclear many-body Hamiltonian through the so-called

\footnotetext{
*Correspondence: mengj@pku.edu.cn

† Jie Meng and Pengwei Zhao equally contributed to this work.

State Key Laboratory of Nuclear Physics and Technology, School of Physics,

Peking University, 100871 Beijing, China
}

effective configuration interactions defined in a given configuration space, and the Hamiltonian is solved in this model space. Such methods are usually applied to medium mass nuclei due to the limitation of the model space. In nuclear DFTs, the ground-state energy of a nucleus is written as a functional of the nucleon density. Starting from the energy density functional, the complicated manybody problem is mapped onto a single-particle problem through the variational principle and, thus, becomes relatively easy to solve. The DFTs are applicable for nuclei all over the nuclide chart. The disadvantage of nuclear DFTs is that, in the first place, it is limited to nuclear ground states. For nuclear spectroscopic properties, one should develop proper extensions based on the energy density functionals.

The covariant DFTs take Lorentz symmetry into account in a self-consistent way and provide an efficient description of nuclei with the underlying large scalar and vector fields of the order of a few hundred $\mathrm{MeV}$, which are hidden in the non-relativistic DFTs. This is clearly seen in the non-relativistic reduction of CDFT via a similarity renormalization method, which provides a possible means to bridge the relativistic and non-relativistic DFTs [5]. As a result, the relativistic framework can provide a good description of nuclear matter and finite nuclei using the Brueckner techniques with only the two-nucleon bare interactions [6]. In the non-relativistic framework, however, three-nucleon interactions, which are still very
Springer

(c) The Author(s). 2021 Open Access This article is licensed under a Creative Commons Attribution 4.0 International License, which permits use, sharing, adaptation, distribution and reproduction in any medium or format, as long as you give appropriate credit to the original author(s) and the source, provide a link to the Creative Commons licence, and indicate if changes were made. The images or other third party material in this article are included in the article's Creative Commons licence, unless indicated otherwise in a credit line to the material. If material is not included in the article's Creative Commons licence and your intended use is not permitted by statutory regulation or exceeds the permitted use, you will need to obtain permission directly from the copyright holder. To view a copy of this licence, visit http://creativecommons.org/licenses/by/4.0/. 
uncertain, have to be introduced for a reasonable description of nuclear matter and/or finite nuclei.

In CDFT, nucleons are described by a Dirac spinor field, which automatically provides the spin degree of freedom. This is of particular importance for nuclear physics, since the spin-orbit interaction is very large for nuclear systems as compared with that in Coulomb systems. In non-relativistic density functionals, one needs additional terms and parameters accounting for the spin-orbit interactions. Moreover, the hidden pseudo-spin symmetry in the single-particle spectrum of nuclei can also be naturally explained in relativistic models [7].

In covariant density functionals, the time-odd parts of the potentials are determined by the currents of nucleons, i.e., the spatial-like components of the vector fields, which have the same coupling constant as the time-like components due to the Lorentz symmetry. Therefore, no new parameters are required for the time-odd fields in the covariant formulations, in spite of the fact that only timeeven fields (time-like components of the vector fields) can be determined by adjusting the nuclear density functionals to experimental ground-state properties. In the non-relativistic density functionals, however, the timeodd fields show some ambiguity. The consistent treatment of time-odd fields in the CDFT is in particular important for describing spectroscopic properties associated with nuclear rotations $[8,9]$.

Due to these advantages, CDFT has received wide attentions over the past decades and achieved a successful description of a large number of nuclear phenomena [1]. In the last decades, numerous efforts have been devoted to developing CDFT based on the finite-range mesonexchange and the zero-range point-coupling models. The CDFT has been successfully applied to describe a large variety of nuclear phenomena from finite nuclei to nuclear matter, from stable nuclei to extremely unstable ones, from spherical nuclei to abnormally deformed ones, from nuclear ground states to excited ones, and from normal nuclear matter to hypernuclear matter with strangeness degrees of freedom. Moreover, the CDFT has also been widely used to investigate the equation of state for compact stars and provides important observables for nucleosynthesis in the astrophysical environment.

The practical usefulness of the nuclear DFTs depends entirely on whether an accurate energy density functional can be found or not. So far, all successful density functionals are phenomenological and are determined by adjusting to the ground-state properties of some selected nuclei. Over the years, several universal energy density functionals have been constructed, providing a successful description of many nuclear ground-state properties with only a few parameters. However, how to build density functionals with the bare nucleon-nucleon interactions is still an open question.

\section{Concept of covariant density functional theory}

The DFT is based on the original theorem of Hohenberg and Kohn [10] which proves that for a many-particle system, the ground-state density constitutes a "basic variable," i.e., all properties of the system, in particular, its ground-state energy, can be written as unique functionals of the ground-state density. Moreover, the ground-state density can be determined from the ground-state energy functional via the variational principle with respect only to the density.

The practical usefulness of the DFT depends entirely on whether an accurate energy density functional can be found or not. The Kohn-Sham DFT [11] provides a very useful way to build the energy density functional by mapping the interacting many-body system onto a fictitious noninteracting system, whose density is assumed to be the true ground-state density. Application of the Hohenberg and Kohn theorem to the fictitious noninteracting system leads to the self-consistent Kohn-Sham equation, which is a single-particle equation. To date, most practical applications of density functional theory use the Kohn-Sham scheme because it allows the appearance of shell effects, which are an essential feature for many quantum mechanical systems. Formally, the solution of the Kohn-Sham DFT looks similar to the Hartree-Fock method. However, it avoids the complicated non-local Fock-term and deals only with local quantities. The complicated integrodifferential equations of Hartree-Fock theory are replaced with simple differential equations, i.e., the Kohn-Sham equation.

There are typically two possible ways to build the energy density functional for many-body systems. One is from the so-called first principles by incorporating known exact constraints, and this has been followed with great success in Coulomb systems due to the well-known Coulomb interaction. For nuclear systems, however, the nucleonnucleon interactions are very complicated. Therefore, one usually resorts to the other way, i.e., to develop an empirical energy density functional with the parameters optimized by fitting to selected data. This way has also been used in the early days of DFT in Coulomb systems.

Nuclear CDFT can be traced back to the relativistic mean field (RMF) model developed by Walecka and Serot in the 1970s [12], where a nucleus is regarded as a composition of a certain number of Dirac nucleons, and the interaction between two nucleons is conveyed by exchanging mesons (see the left diagram of Fig. 1). For a satisfying description of both nuclear matter and finite nuclei, the scalar $\sigma$ meson, vector $\omega$ meson, and isovector-vector $\rho$ meson are considered. Moreover, it is convenient to establish a point-coupling density functional by an analogy with the meson-exchange RMF models. Specifically, one can replace an exchanged meson in the RMF models by a contact interaction between nucleons in every channel of the 


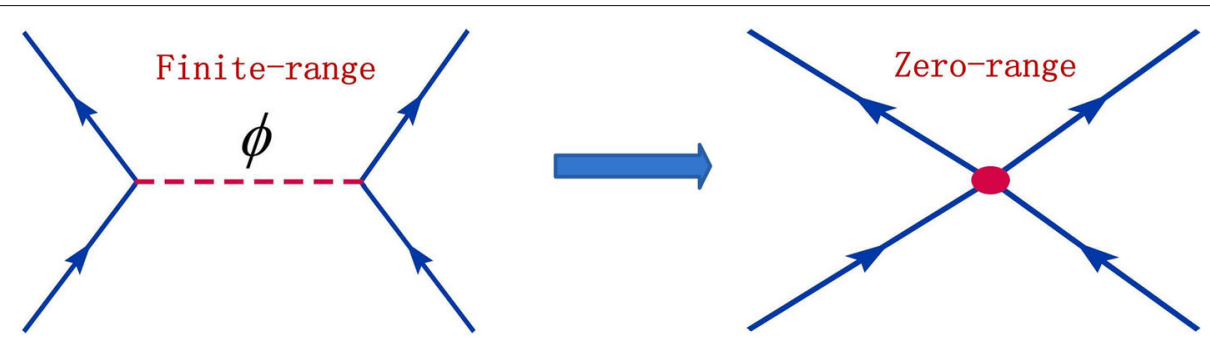

Fig. 1 Finite-range (left) and zero-range (right) nucleon-nucleon interactions employed in building the nuclear covariant density functional

interactions, e.g., the scalar, vector, and isovector channels (see the right diagram of Fig. 1). In such a way, the successful RMF models can be reformulated in the framework of Kohn-Sham DFT.

The essential ingredient of CDFT is the relativistic Kohn-Sham equation, which has the form of a singleparticle Dirac equation,

$$
[\boldsymbol{\alpha} \cdot(-i \nabla-\boldsymbol{V})+V+\beta(m+S)] \psi_{k}=\varepsilon_{k} \psi_{k} .
$$

Here, the scalar $S(\boldsymbol{r})$ and vector $V^{\mu}(\boldsymbol{r})$ potentials are connected with various densities and currents in a selfconsistent way.

Nuclei are self-bound systems, so there is no external potential. This is an important difference between DFT in Coulomb systems and in nuclear ones. The HohenbergKohn theorem was derived under the assumption that the system is confined by an external potential. Due to the translational invariance of a self-bound nucleus, the exact density in the laboratory frame has to be a constant, and the corresponding Hohenberg-Kohn theorem with this constant density is useless. On the other hand, it has been shown that the Hohenberg-Kohn theorem holds for the intrinsic density depending on the center-of-mass coordinate in the intrinsic frame. A possible justification for the functional of the wave-packet density produced by the spontaneous breaking of symmetries in finite systems was given in Ref. [13]. The argument is exact for the translational symmetry breaking since the center-of-mass motion and the intrinsic motion are exactly decoupled, while it is approximate for the rotational symmetry breaking of deformed nuclei.

With the DFT, in principle, we can calculate only the exact local density and the quantities related to it, such as the total energy and expectation values of local onebody operators including radii, quadrupole moments, etc. With the intrinsic density, one needs to transform to the laboratory frame from the intrinsic frame by, for example, projection techniques, which are usually connected with approximations. For nuclei with relatively flat energy surfaces in the deformation space, one has to take fluctuations with different deformations into account. These methods go definitely beyond the mean-field concept, but have been turned out to be very successful [14].
The static DFT can also be extended to the timedependent case for describing nuclear dynamic processes with the Runge-Gross theorem [15], which provides an exact mapping of the full time-dependent manybody problem onto a time-dependent single-particle problem. Such extensions in the framework of nonrelativistic DFT have been developed, and with the everimproving computational capabilities, the unrestricted three-dimensional calculations with modern nuclear density functionals become available. For CDFT, however, an unrestricted three-dimensional calculation is not easy because of the variational collapse problem in solving the Dirac equation in the coordinate space, which is induced by the states in the Dirac sea. It is only available recently in Ref. [16], where the time-dependent covariant density functional theory in 3D lattice space has been developed for the first time.

\section{Global description of nuclear landscape}

A global study of nuclear masses associated with the beyond mean-field correlations in CDFT has been carried out with PC-PK1 [17] using a collective Hamiltonian method [18]. A large-scale deformation constrained CDFT calculation was performed to obtain the parameters of the collective Hamiltonian, which describes the beyond mean-field correlations from quantum vibrations and rotations. Then, the nuclear ground states are obtained by solving the collective Hamiltonian.

Figure 2 depicts the mass differences between the corresponding PC-PK1 predictions and the data for 575 eveneven nuclei [19]. No more parameters beyond the PC-PK1 density functional have been introduced in the calculations. The root-mean-square mass deviation is $1.14 \mathrm{MeV}$, which is, so far, the best global description of nuclear masses in DFT.

Due to the successful description of nuclear masses with PC-PK1, it has also been used to explore the nuclear landscape with $8 \leq Z \leq 120$ from the proton drip line to the neutron drip line with the relativistic continuum HartreeBogoliubov (RCHB) theory [20], in which the continuum effects are treated properly. By assuming the spherical symmetry, 9035 nuclei are predicted to be bound, and it was found that the coupling between the bound states 


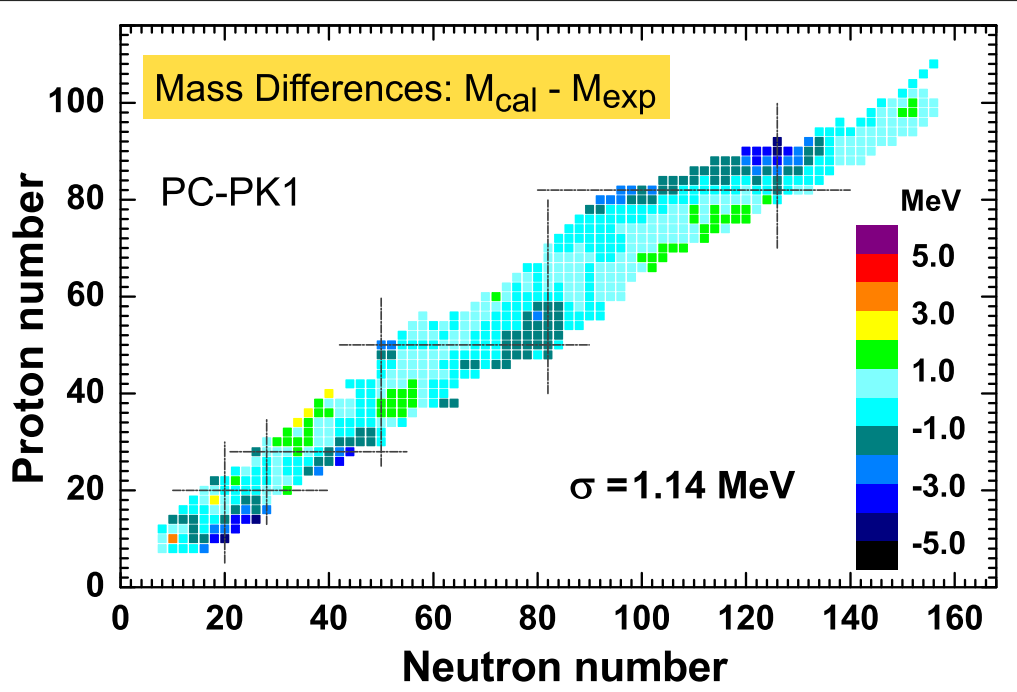

Fig. 2 (Color online) Mass differences between the PC-PK1 predictions and the data for 575 even-even nuclei [19]. The $\sigma$ value represents the root-mean-square deviations between the theoretical predictions and data. Taken from Ref. [18]

and the continuum due to the pairing correlations plays an essential role in extending the nuclear landscape. In Fig. 3, it is compared to the predicted number of possible isotopes from the average of the DFT predictions and the anticipated range of isotopes to be available at Facility for Rare Isotope Beams (FRIB) up to uranium. It is quite encouraging to consider the deformation and continuum effects simultaneously in determining the drip line in the future. Such calculations are more challenging, but are feasible in the framework of the deformed relativistic Hartree-Bogoliubov theory in continuum [21], and the recent progress can be found in Ref. [22].

\section{Nuclear chiral rotation}

Novel rotational excitations like the magnetic and antimagnetic rotations observed in nearly spherical nuclei and the chiral rotation in triaxial nuclei have attracted a lot of attention. Unlike the conventional rotation, here the rotational axis does not necessarily coincide with any principal axis of nuclear density distribution and, thus, a tilted axis cranking is mandatory to describe them self-consistently in the framework of CDFT.

Chirality is a well-known phenomenon in science, such as chemistry, biology, molecular and particle physics, etc. In nuclear physics, chirality was originally suggested by Frauendorf and Meng in 1997 [23], and its experimental signal is the so-called chiral doublet bands, which consist of a pair of nearly degenerate bands with the same parity. The chirality is not restricted to a specific configuration in a certain mass region. The resultant possibility of having more than one pair of chiral bands in a single nucleus, i.e., multiple chiral doublet bands $(\mathrm{M} \chi \mathrm{D})$, was demonstrated by searching for triaxial chiral configurations in $\mathrm{Rh}$ isotopes using constrained relativistic mean-field calculations in Ref. [24]. The M $\chi \mathrm{D}$ in ${ }^{106} \mathrm{Rh}$ was further studied with the three-dimensional tilted axis cranking CDFT in a fully self-consistent and microscopic way [25], and two distinct sets of chiral solutions with negative and positive parities, respectively, were found.

In the experiment, a pair of negative-parity chiral bands has been observed in ${ }^{106} \mathrm{Rh}$. In Fig. 4, the calculated rotational excitation energies and rotational frequencies $\hbar \omega$ for the observed chiral bands in ${ }^{106} \mathrm{Rh}$ are shown in comparison with data. It can be clearly seen in the upper panel that the experimental excitation energies of band 1 (the lower band) can be reproduced well. Due to the nature of a mean-field approach, it does not take into account either the chiral vibrations or the tunneling between the leftand right-handed chiral sectors. Therefore, the energy splitting between the two partner bands cannot be seen.

The chiral geometry is seen in the CDFT by examining the angular momentum geometry, and its evolution is reflected in the lower panel of Fig. 4, where the experimental rotational frequencies $\hbar \omega$ for the two partner bands behave very similarly with respect to the angular momentum, and they agree with the calculated results. In particular, the data indicate a kink around $I=12 \hbar$, where the slope of the $\hbar \omega$ vs spin curve changes abruptly. In comparison with the calculated results, the appearance of this kink is due to the change from the planar to the chiral solutions around $\hbar \omega \sim 0.46 \mathrm{MeV}$. In the planar regime, the angular momentum is generated in the plane spanned by the short and long axes, while in the chiral one, it increases along the intermediate axis, which has larger moments of inertia. 


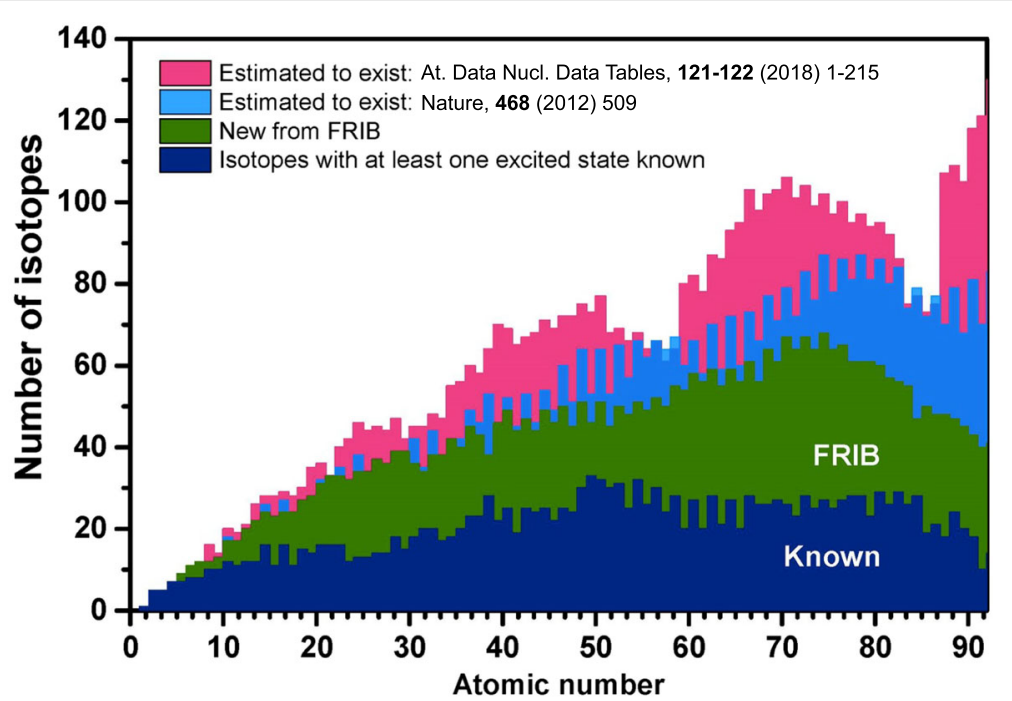

Fig. 3 (Color online) Number of isotopes of elements up to uranium estimated to exist in theoretical predictions and to be produced at the Facility for Rare Isotope Beams (FRIB) in comparison with the known ones

\section{Nuclear dynamics}

Apart from the static properties of nuclei, nuclear dynamic processes, such as scattering, fission, and fusion, have also been at the frontiers of nuclear physics. To study nuclear dynamic processes, one has to extend the static
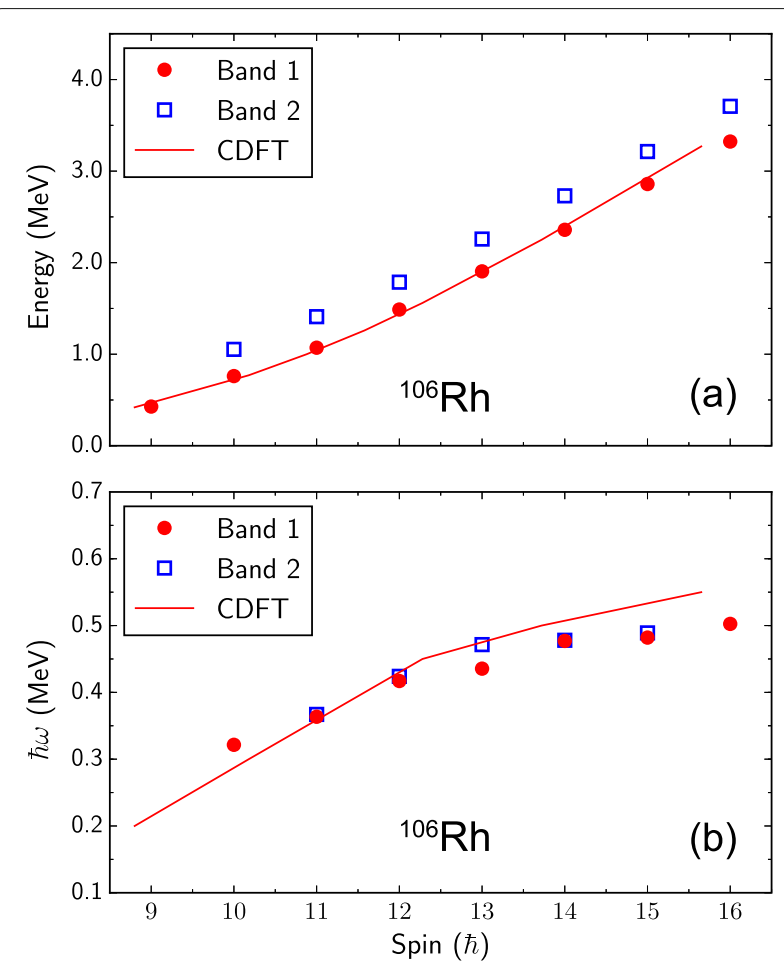

Fig. 4 Calculated rotational excitation energies (top) and rotational frequencies $\hbar \omega$ (bottom) for the observed chiral bands in ${ }^{106} \mathrm{Rh}$, in comparison with data. Taken from [25]
CDFT to the time-dependent CDFT. In Ref. [16], the timedependent CDFT in 3D lattice space has been developed and applied to investigate the microscopic dynamics of the linear-chain cluster states in carbon isotopes by simulating the ${ }^{4} \mathrm{He}+{ }^{8} \mathrm{Be}$ and ${ }^{4} \mathrm{He}+{ }^{10} \mathrm{Be}$ resonant scatterings without any symmetry assumptions.

In Fig. 5, four snapshots from the long time evolution of the two collisions at the impact parameter $b=0.1$ $\mathrm{fm}$ are presented in terms of the total density distributions at the times $t=100,600,1200$, and $1800 \mathrm{fm} / c$. For the ${ }^{4} \mathrm{He}+{ }^{8} \mathrm{Be}$ collision, the system retains the linear-chain configuration up to the time at about $745 \mathrm{fm} / c$, and then it starts to bend and forms a more compact shape at longer times [see Fig. $5 \mathrm{c}$ and d]. For the ${ }^{4} \mathrm{He}+{ }^{10} \mathrm{Be}$ collision, however, the linear-chain configuration of the system can be retained at the time up to $1200 \mathrm{fm} / c$. Moreover, comparing Fig. $5 \mathrm{f}$ and $\mathrm{g}$, the positions of the $\alpha$-like and ${ }^{10} \mathrm{Be}$-like clusters could be exchanged during the time evolution, which reflects the quasiperiodic oscillating motion of the $\alpha$-like and ${ }^{10} \mathrm{Be}$-like clusters. In Ref. [16], it was found that the valence neutrons slow down the longitudinal quasiperiodic oscillations of the clusters, i.e., dynamical isospin effects, and this is responsible for the much longer survival time of the linear-chain states in the ${ }^{4} \mathrm{He}+{ }^{10} \mathrm{Be}$ collision.

\section{Relativistic ab initio calculations}

In recent years, the connection between covariant density functionals and realistic nucleon-nucleon interactions has become a hot topic. As the basic theory of the strong interaction, quantum chromodynamics (QCD) and its chiral symmetry are being used for nuclear structure in the region of low momenta. The non-relativistic form of the bare nucleon-nucleon forces is expressed in terms 


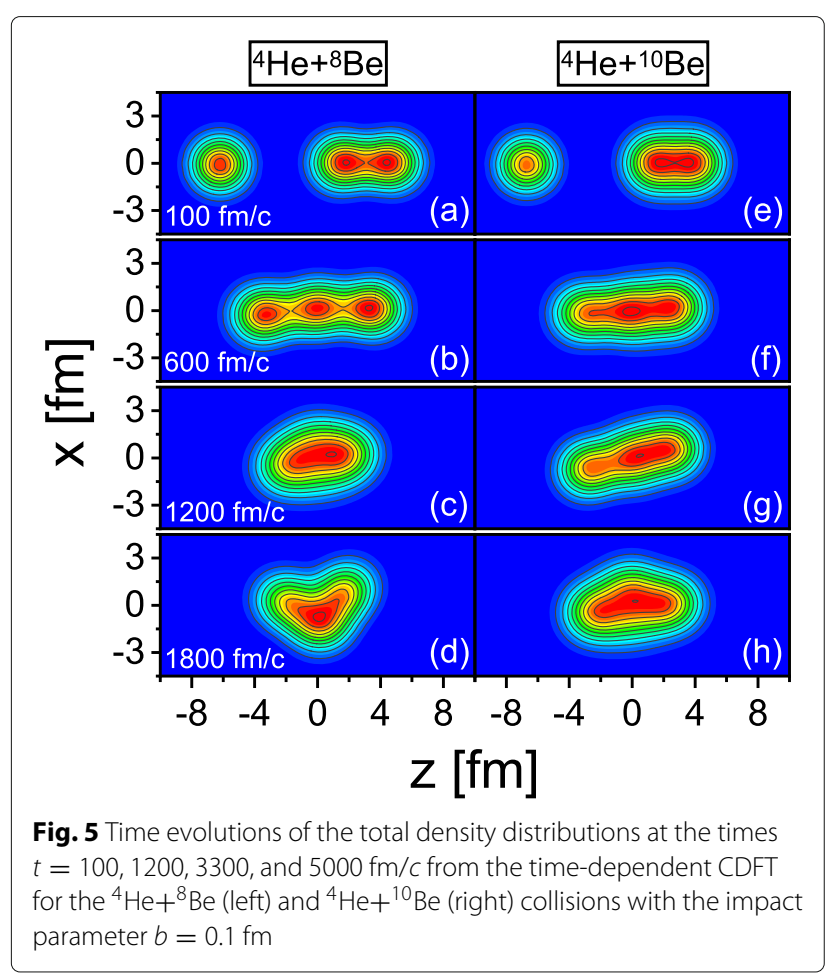

of chiral perturbation theory at various orders. At a long and intermediate distance, the pion meson resulting from the breaking of chiral symmetry plays an essential role, while the short-range part of the force, acting at a scale not influencing directly the details of nuclear physics, is written in terms of various renormalization parameters. At present, these chiral forces still contain a large number of phenomenological parameters, which are adjusted to the experimental nucleon-nucleon scattering data. In the next step, sophisticated numerical techniques have been developed to solve the nuclear many-body problem in terms of these chiral forces. This is possible nowadays for light nuclei. However, the results are a bit disappointing and agreement with experimental binding energies, radii, and spectra of finite nuclei can be achieved only by introducing bare three-body forces containing additional phenomenological parameters adjusted to light nuclei.

On the other hand, the fully self-consistent relativistic Brueckner-Hartree-Fock (RBHF) theory, without any adjustable parameters, has been successful even at the Hartree-Fock level, using only the two-body realistic interactions fitted to the nucleon-nucleon scattering and deuteron properties. In Fig. 6, the energy per nucleon and the charge radius of ${ }^{16} \mathrm{O}$ calculated by the RBHF theory are shown in comparison with the renormalized Brueckner-Hartree-Fock (BHF) and the relativistic effective density approximation (EDA) results. The relativistic effects improve the description considerably, both for the energy and the radius. By comparing the results of EDA

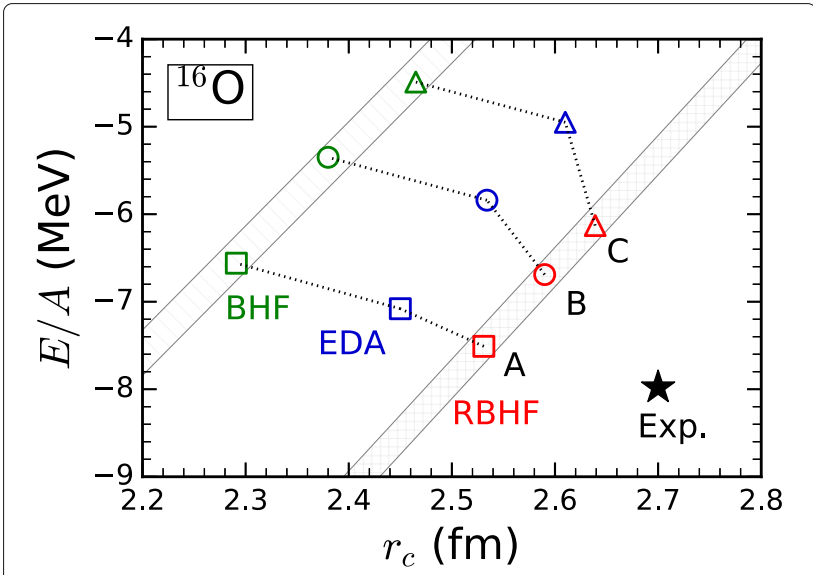

Fig. 6 Energy per nucleon $E / A$ for ${ }^{16} \mathrm{O}$ as a function of the charge radius $r_{c}$ calculated by the relativistic Brueckner-Hartree-Fock (RBHF) using the interactions Bonn $A, B$, and $C$, in comparison with the results given by the Brueckner-Hartree-Fock (BHF) and the relativistic effective density approximation (EDA). Taken from Ref. [6]

and RBHF, it reveals that the self-consistency is important to improve the description. Certain rearrangement effects brought by adopting renormalized occupation probabilities in RBHF may further improve the description for finite nuclei, especially for the radii.

\section{Summary and perspectives}

Over the past decades, the relativistic density functional theory has been greatly developed and widely applied to investigate a variety of nuclear phenomena. We briefly review the concept of covariant density functional theory in nuclear physics and several applications in describing nuclear ground-state and excitation properties as well as nuclear dynamics. There are still many interesting topics and open problems in this field. One of the most urgent issues is the inclusion of the tensor-force effects in the covariant density functionals. There has been several works on this topic within the relativistic Hartree-Fock framework [26-28], while it is very complex and time consuming for even the ground state of deformed nuclei. Another important related issue is on the determination of the tensor-force strength. It is usually difficult to fix the tensor terms of the functional by experimental data because of their strong interplay with other terms. In this sense, the ab initio RBHF calculations can provide essential information to guide the construction of DFTs. For example, the difficulty of nuclear DFTs to fix the tensor terms of the functional by experimental data may be solved by nuclear ab initio calculations for a simple ideal system, i.e., neutron drops. Moreover, ab initio calculations of the nuclear equation of state (EOS) are accessible over a wide range of nucleon densities and are far beyond the densities reachable in the laboratory. Therefore, in 
addition to experimental data, the ab initio results at supra- and subsaturation densities may also need to be considered in building a universal density functional. This also induces a problem of exploring the proper density dependence in the different terms of the functional. In this sense, the ab initio calculations are very important to build a universal density functional.

\section{Acknowledgements}

This work was partly supported by the National Key R\&D Program of China (Contract Nos. 2018YFA0404400 and 2017YFE0116700), the National Natural Science Foundation of China (Grant Nos. 11621131001, 11875075, 11935003, and 11975031), and the State Key Laboratory of Nuclear Physics and Technology, Peking University (No. NPT2020ZZ01).

\section{Authors' contributions}

The authors read and approved the final manuscript.

\section{Competing interests}

The authors declare that they have no competing interests.

Received: 7 September 2020 Accepted: 16 November 2020

Published online: 05 February 2021

\section{References}

1. J. Meng (ed.), Relativistic Density Functional for Nuclear Structure (World Scientific), (2015). https://doi.org/10.1142/9872

2. B. R. Barrett, P. Navratil, J. P. Vary, Ab initio no core shell model. Prog. Part. Nucl. Phys. 69, 131 (2013)

3. J. Carlson, S. Gandolfi, F. Pederiva, S. C. Pieper, R. Schiavilla, K. E. Schmidt, R. B. Wiringa, Quantum Monte Carlo methods for nuclear physics. Rev. Mod. Phys. 87, 1067 (2015)

4. E. Caurier, G. Martínez-Pinedo, F. Nowacki, A. Poves, A. P. Zuker, The shell model as a unified view of nuclear structure. Rev. Mod. Phys. 77, 427 (2005)

5. Z.X. Ren, P. W. Zhao, Toward a bridge between relativistic and nonrelativistic density functional theories for nuclei. Phys. Rev. C. 102, 021301(R) (2020)

6. S. Shen, H. Liang, W. H. Long, J. Meng, P. Ring, Towards an ab initio covariant density functional theory for nuclear structure. Prog. Part. Nucl. Phys. 109, 103713 (2019)

7. H. Liang, J. Meng, S.-G. Zhou, Hidden pseudospin and spin symmetries and their origins in atomic nuclei. Phys. Rep. 570, 1 (2015)

8. J. Meng, J. Peng, S.-Q. Zhang, P.-W. Zhao, Progress on tilted axis cranking covariant density functional theory for nuclear magnetic and antimagnetic rotation. Front. Phys. 8, 55 (2013)

9. D. Vretenar, A. V. Afanasjev, G. A. Lalazissis, P. Ring, Relativistic Hartree Bogoliubov theory: static and dynamic aspects of exotic nuclear. Phys. Rep. 409, 101 (2005)

10. P. Hohenberg, W. Kohn, Inhomogeneous Electron Gas. Phys. Rev. 136 B864 (1964)

11. W. Kohn, L. J. Sham, Self-Consistent Equations Including Exchange and Correlation Effects. Phys. Rev. 140, A1133 (1965)

12. B. D. Serot, J. D. Walecka, The Relativistic Nuclear Many-Body Problem. Adv. Nucl. Phys. 16, 1 (1986)

13. T. Nakatsukasa, K. Matsuyanagi, M. Matsuo, K. Yabana, Time-dependent density-functional description of nuclear dynamics. Rev. Mod. Phys. 88, 045004 (2016)

14. T. Nikšić, D. Vretenar, P. Ring, Relativistic nuclear energy density functionals: Mean-field and beyond. Prog. Part. Nucl. Phys. 66, 519 (2011)

15. E. Runge, E.K.U., Density-Functional Theory for Time-Dependent Systems. Gross. Phys. Rev. Lett. 52, 997 (1984)

16. Z. Ren, P. Zhao, J. Meng, Dynamics of the linear-chain alpha cluster in microscopic time-dependent relativistic density functional theory. Phys. Lett. B. 801, $135194(2020)$

17. P. W. Zhao, Z. P. Li, J. M. Yao, J. Meng, New parametrization for the nuclear covariant energy density functional with a point-coupling interaction. Phys. Rev. C. 82, 054319 (2010)
18. K. Q. Lu, Z. X. Li, Z. P. Li, J. M. Yao, J. Meng, Global study of beyond-mean-field correlation energies in covariant energy density functional theory using a collective Hamiltonian method, vol. 91, (2015), p. 027304

19. M. Wang, G. Audi, A. Wapstra, F. Kondev, M. MacCormick, X. Xu, B. Pfeiffer, The Ame2012 atomic mass evaluation. Chin. Phys. C. 36, 1603 (2012)

20. X. Xia, Y. Lim, P. Zhao, H. Liang, X. Qu, Y. Chen, H. Liu, L. Zhang, S. Zhang, Y. Kim, J. Meng, The limits of the nuclear landscape explored by the relativistic continuum Hartree-Bogoliubov theory. At. Data Nucl. Data Tables. 121-122, 1 (2018)

21. S.-G. Zhou, J. Meng, P. Ring, E.-G. Zhao, Neutron halo in deformed nuclei. Phys. Rev. C. 82, 011301 (R) (2010)

22. K. Zhang, M.-K. Cheoun, Y.-B. Choi, P. S. Chong, J. Dong, L. Geng, E. Ha, X He, C. Heo, M. C. Ho, E. J. In, S. Kim, Y. Kim, C.-H. Lee, J. Lee, Z. Li, T. Luo, J. Meng, M.-H. Mun, Z. Niu, C. Pan, P. Papakonstantinou, X. Shang, C. Shen, G. Shen, W. Sun, X.-X. Sun, C. K. Tam, C. Thaivayongnou, S. H. Wang, X. Wong, Y. Xia, R. Yan, W.-Y. Yeung, T. C. Yiu, S. Zhang, W. Zhang, S.-G. Zhou, Deformed relativistic Hartree-Bogoliubov theory in continuum with a point-coupling functional: Examples of even-even $\mathrm{Nd}$ isotopes. Phys. Rev. C. 102, $024314(2020)$

23. S. Frauendorf, J. Meng, Tilted rotation of triaxial nuclei. Nucl. Phys. A. 617, 131 (1997)

24. J. Meng, J. Peng, S. Q. Zhang, S.-G. Zhou, Possible existence of multiple chiral doublets in Rh-106. Phys. Rev. C. 73, 037303 (2006)

25. P. W. Zhao, Multiple chirality in nuclear rotation: A microscopic view. Phys Lett. B. 773, 1 (2017)

26. W. H. Long, H. Sagawa, N. Van Giai, J. Meng, Shell structure and rho-tensor correlations in density dependent relativistic Hartree-Fock theory. Phys. Rev. C. 76, 034314 (2007)

27. L. J. Jiang, S. Yang, B. Y. Sun, W. H. Long, H. Q. Gu, Nuclear tensor interaction in a covariant energy density functional. Phys. Rev. C. 91, 034326 (2015)

28. Z. Wang, Q. Zhao, H. Liang, W. H. Long, Quantitative analysis of tensor effects in the relativistic Hartree-Fock theory. Phys. Rev. C. 98, 034313 (2018)

\section{Publisher's Note}

Springer Nature remains neutral with regard to jurisdictional claims in published maps and institutional affiliations.

\section{Submit your manuscript to a SpringerOpen ${ }^{\odot}$ journal and benefit from:}

- Convenient online submission

- Rigorous peer review

- Open access: articles freely available online

- High visibility within the field

- Retaining the copyright to your article

Submit your next manuscript at $>$ springeropen.com 